(C) 2018 IEEE. Personal use of this material is permitted. Permission from IEEE must be obtained for all other uses, in any current or future media, including reprinting/republishing this material for advertising or promotional purposes, creating new collective works, for resale or redistribution to servers or lists, or reuse of any copyrighted component of this work in other works. 


\title{
Decentralized Two-Channel Active Noise Control for Single Frequency by Shaping Matrix Eigenvalues
}

\author{
Guoqiang Zhang, Jiancheng Tao, Xiaojun Qiu, and Ian Burnett
}

\begin{abstract}
In an active noise control (ANC) system, computational complexity is one major concern when designing practical control algorithms. For an ANC system with multiple secondary sources and error microphones, one approach to reducing computational complexity is to apply a decentralized control scheme rather than centralized approaches. A decentralized scheme attempts to control a number of small-size ANC subsystems independently. In this paper, we consider decentralized control of a two-channel ANC system tackling a noise disturbance in the frequency domain, where each channel consists of one secondary source and one error microphone. We propose a decentralized control method which is able to achieve the same noise reduction performance as the centralized controller with guaranteed convergence. The key step in designing the control method is to properly shape the eigenvalues of a matrix which models the two-channel secondary paths for each frequency index.
\end{abstract}

Index Terms-Active noise control (ANC), decentralized controller, adaptive controller.

\section{INTRODUCTION}

Active noise control (ANC) techniques have been successfully exploited to remove or mitigate sound noise in many applications, such as designing ANC systems for headphone applications [1], reducing acoustic noise in magnetic resonance (MR) imaging [2], and creating a quiet zone around listener ears [3]. The basic principle of an ANC system is to introduce one or more secondary sources to interfere destructively with the primary sound by using loudspeakers and error microphones.

Three factors are usually considered when designing a practical adaptive control algorithm for an ANC system: namely, guaranteed convergence and the convergence speed, noise reduction performance, and computational complexity. The standard filtered-x LMS algorithm [4] is probably the most popular one in the literature with a reasonable balance among the three factors. The algorithm serves as a foundation for developing more advanced methods for different environmental conditions or system requirements as described below.

For a single-channel ANC system with one secondary source and one error microphone, developing self-adaptive and low-complexity control methods is of primary concern in the ANC community. One promising research trend is to perform

G. Zhang, X. Qiu and I. Burnett are with the Center for Audio, Acoustics and Vibration (CAAV), University of Technology, Sydney, Australia. Email: \{guoqiang.zhang, xiaojun.qiu, Ian.Burnett\}@uts.edu.au.

J. Tao is with Institute of Acoustics, Nanjing University, China. Email: jctao@nju.edu.cn. adaptive noise control by avoiding estimation of the singlechannel secondary path [5], [6], [7]. The motivation behind this approach is that for some applications, the characteristics of the secondary path may vary over time, and further there is no need to obtain an accurate secondary path model for the control methods to work [6]. The basic idea is to roughly estimate the phase of the secondary path (e.g., selection from four directions like $0,90,180,270$ degrees in [6]), and then use the information to adaptively minimize the error signal. The developed methods are more flexible and have low computational complexity.

For a large scale ANC system with multiple secondary sources and error microphones, the standard filtered-x LMS algorithm adjusts the inputs of all secondary sources in response to the outputs of all error microphones [4]. To do so, a central controller is employed to collect and process signals from all the error microphones, and then make proper adjustments, referred to as the centralized control scheme. The system requires a considerable amount of computational power and expensive wiring for the algorithm to work.

In the literature, different approaches have been proposed towards effective control of a large scale ANC system. The work of [8] considers preprocessing the error signals to reduce the computational complexity of the central controller. Instead of processing multiple error signals directly, [8] combines all the error signals into one signal and then uses it for adaptive centralized control. However, high communication load is still required to route signals from all the error microphones to the central controller.

Distributed computation of the control signals is an alternative approach to combating the high computational complexity of centralized control [9], [10], [11]. Specifically, [9] treats an ANC system as a ring network where each secondary source is taken as one node in the network. The computational burden is then distributed across all the secondary sources by performing incremental computation sequentially over the ring network. At each iteration, the computation unit at each secondary source receives, updates and then transmits the control signal of all the secondary sources by interacting with neighbors. The method achieves the same noise reduction performance as the centralized controller at the cost of high transmission bandwidth and delay. The work of [10] extends [9] by utilizing a diffusion strategy in the updating expressions of the control signal.

A decentralized control scheme aims to reduce both the computational complexity and transmission load of the cen- 
tral controller (see [12], [13]). The basic idea is to treat a large scale ANC system as a combination of small-size ANC subsystems. Each subsystem adjusts its own control signal only based on its associated error microphones. Due to the inherent coupling between the secondary sources and error microphones, it is challenging to design effective and robust control methods for the multiple subsystems. The work of [12] proposed and analyzed a feedforward decentralized control method by studying matrix eigenvalues in the frequency domain, where the matrix is determined by transfer functions of the secondary paths of the whole system. It is found that if the inputs of the secondary sources are constrained to be sufficiently small in magnitude, the control method is guaranteed to converge (see Section 3 for detailed information). We note that small inputs implicitly degrade the noise reduction performance, which is practically unattractive. The recent work [13] conducted extensive simulations and experiments for the control method of [12] and drew a number of insightful observations.

In this paper, we consider feedforward decentralized control of a two-channel ANC system, where each channel consists of one secondary source and one error microphone. Our motivation is to build an in-depth understanding of the performance limit for two-channel decentralized control, and gain insights for effective control of a multi-channel ANC system.

To tackle the drawback of degraded noise reduction performance noted in [12], we propose a novel decentralized adaptive control algorithm in the frequency domain. In designing the control algorithm, we first study the eigenvalues of a matrix which models the two-channel secondary path for each frequency bin. Two free parameters are identified, and then used to shape the eigenvalues of the matrix whenever necessary so that the resulting eigenvalues have nonnegative or positive real parts. By doing so, the constraints on the inputs to the secondary sources can be removed or alleviated when applying the steepest descent method, thus achieving (or nearly) the same noise reduction performance as the centralized controller. A procedure to shape the two eigenvalues is provided, which essentially finds the right direction to ensure convergence of the steepest descent method. Simulation results confirm the effectiveness of the proposed decentralized controller.

The remainder of the paper is organized as follows. Section II defines the two-channel signal processing problem in the frequency domain. Section III briefly reviews the conventional decentralized control method in [12] for the two channel case. Based on the results in Section III, we motivate our new decentralized control method in Section IV. Section V presents the simulation results, followed by conclusions drawn in Section VI.

\section{Two-Channel Signal Model}

Consider a primary sound disturbance generated by a point monopole at a single frequency. We exploit an ANC system to mitigate or remove the disturbance in the same environment. The considered ANC system consists of 2 secondary sources and 2 error microphones, where each secondary source and its collocated error microphone form a single-channel ANC subsystem (see Fig. 1). As the considered disturbance is from
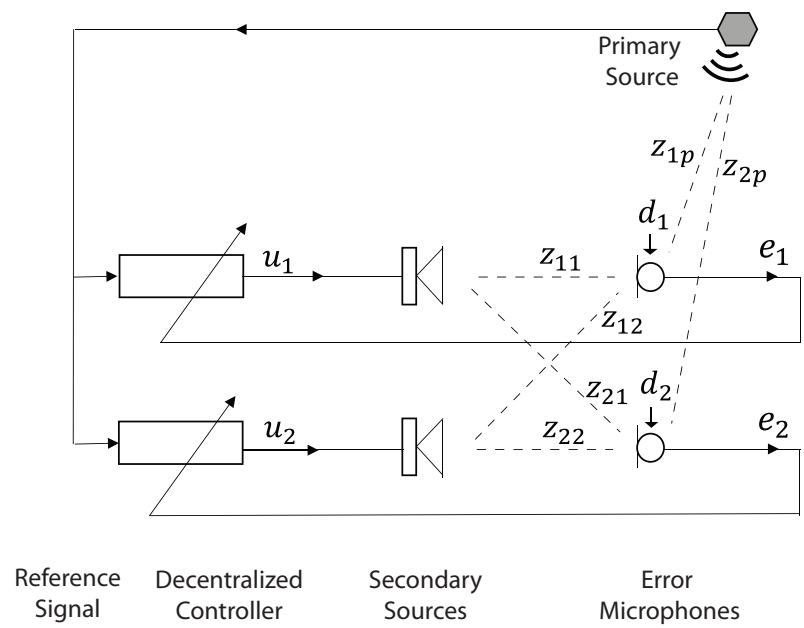

Fig. 1. Demonstration of a decentralized two-channel ANC system, where each subsystem consists of a secondary source and its collocated error microphone. The parameter $z_{v s}, v=1,2, s=p, 1,2$, denotes the frequency response of the acoustic path from $s$ to $v$.

one frequency, all the signals can be represented as scalar complex numbers. We omit the frequency index here for concision.

Based on Fig. 1, the complex steady state signal at the $i$ th error microphone can be represented as

$$
e_{i}=d_{i}+z_{i j} u_{j}+z_{i i} u_{i} \quad i, j=1,2,
$$

where $d_{i}$ represents the disturbance from the primary source at the $i$ th error microphone, $u_{i}$ (or $u_{j}$ ) is the complex input to the $i$ th (or $j$ th) secondary source, and $z_{i j}$ represents the complex response from the $j$ th secondary source to the $i$ th error microphone. Eq. (1) can be further expressed into a compact vector form as

$$
\boldsymbol{e}=\boldsymbol{d}+\boldsymbol{Z} \boldsymbol{u}, \text { where } \boldsymbol{Z}=\left[\begin{array}{ll}
z_{11} & z_{12} \\
z_{21} & z_{22}
\end{array}\right] .
$$

The $2 \times 2$ complex matrix $\boldsymbol{Z}$ captures the joint response of the two secondary sources at the two error microphones.

The objective of a controller is to minimize the error vector $\boldsymbol{e}$ by properly adjusting the input vector $\boldsymbol{u}$. For the case that $\boldsymbol{Z}$ is nonsingular, the optimal input vector $\boldsymbol{u}^{\star}$ is computed by minimizing the sum of squared error signals directly, given by [12]

$$
\begin{aligned}
\boldsymbol{u}^{\star} & =\arg \min _{\boldsymbol{u}}\left(\frac{1}{2} \boldsymbol{e}^{H} \boldsymbol{e}\right) \\
& =-\left(\boldsymbol{Z}^{H} \boldsymbol{Z}\right)^{-1} \boldsymbol{Z}^{H} \boldsymbol{d} \\
& =-\boldsymbol{Z}^{-1} \boldsymbol{d},
\end{aligned}
$$

where the superscripts $(\cdot)^{H}$ and $(\cdot)^{-1}$ denote conjugate transpose and matrix inversion, respectively.

When the matrix $Z$ is singular, a common strategy for computing $\boldsymbol{u}^{\star}$ is to minimize the sum of squared error signals plus a quadratic penalty function on $\boldsymbol{u}$, given by [12]

$$
\begin{aligned}
\boldsymbol{u}^{\star} & =\arg \min _{\boldsymbol{u}}\left(\frac{1}{2} \boldsymbol{e}^{H} \boldsymbol{e}+\frac{\beta}{2} \boldsymbol{u}^{H} \boldsymbol{u}\right) \\
& =-\left(\boldsymbol{Z}^{H} \boldsymbol{Z}+\beta \boldsymbol{I}\right)^{-1} \boldsymbol{Z}^{H} \boldsymbol{d},
\end{aligned}
$$


where $\beta>0$ is a positive weighting factor, and $\boldsymbol{I}$ is the identity matrix. The factor $\beta$ ensures that resulting matrix $\boldsymbol{Z}^{H} \boldsymbol{Z}+\beta \boldsymbol{I}$ is positive definite, and thus is nonsingular.

It is known that a centralized controller is able to converge to the optimal control signal (3) or (4) after a sufficient number of iterations by following the steepest decent algorithm [12]. Our objective is to propose a new decentralized control method that is able to converge to (3) or (4), thus achieving the same noise reduction performance as the centralized controller.

\section{The Conventional Decentralized Adaptive CONTROL METHOD}

In this section, we briefly review the analysis results of the conventional decentralized control method presented in [12]. Our main motivation is to identify the limitations of the conventional method and serve as a preliminary work to motivate our new method in next section.

The work of [12] considered an ANC system consisting of $M$ decentralized controllers, one for each single-channel subsystem. In the following, we only present the results for the two-channel case for concision. From [12], the $l$ th (i.e., $l=1,2$ ) controller attempts to adjust the input $u_{l}$ to its own secondary source in response to the error signal $e_{l}$ only. To combat the interference from other secondary sources, a quadratic penalty function of the input $u_{l}$ is introduced, which is similar to (4). The resulting objective function for the $l$ th controller is thus given by

$$
J_{l}\left(u_{l}\right)=\frac{1}{2} e_{l}^{*} e_{l}+\frac{\beta}{2} u_{l}^{*} u_{l} \quad l=1,2,
$$

where the superscript $*$ represents the conjugate operation. The gradient of $J_{l}$ w.r.t. $u_{l}$ can then be computed as [12]

$$
\nabla_{u_{l}} J_{l}=z_{l l}^{*} e_{l}+\beta u_{l} \quad l=1,2 .
$$

Upon obtaining the gradient expression (6), the input to the $l$ th secondary source can be adaptively controlled using the steepest descent algorithm as

$$
\begin{aligned}
u_{l}(k+1) & =u_{l}(k)-\mu \nabla_{u_{l}} J_{l}(k) \\
& =u_{l}(k)-\mu z_{l l}^{*} e_{l}(k)-\mu \beta u_{l}(k) \quad l=1,2,
\end{aligned}
$$

where $k$ is the iteration index and $\mu>0$ is the step size. Rewriting (7) into a compact vector form produces

$$
\boldsymbol{u}(k+1)=(1-\mu \beta) \boldsymbol{u}(k)-\mu \boldsymbol{Z}_{d}^{H} \boldsymbol{e}(k),
$$

where $\boldsymbol{Z}_{d}=\operatorname{diag}\left(\left[z_{11}, z_{22}\right]\right)$ is a diagonal matrix of its vector. Plugging the expression (2) for $\boldsymbol{e}$ at iteration $k$ into (8) yields

$$
\boldsymbol{u}(k+1)=\left[\boldsymbol{I}-\mu\left(\beta \boldsymbol{I}+\boldsymbol{Z}_{d}^{H} \boldsymbol{Z}\right)\right] \boldsymbol{u}(k)-\mu \boldsymbol{Z}_{d}^{H} \boldsymbol{d},
$$

where the two parameters $\mu$ and $\beta$ remain to be specified. The iterates in (9) converge to a fixed point if and only if all the eigenvalues of the matrix in the square bracket have modulus less than one. It is not difficult to conclude that as long as the real parts of all the eigenvalues of $\beta \boldsymbol{I}+\boldsymbol{Z}_{d}^{H} \boldsymbol{Z}$ are positive, a sufficient small step size $\mu$ would allow the iterates in (9) to converge. The problem boils down to how to select the parameter $\beta$ towards guaranteed convergence.

Theoretical analysis on the selection of $\beta$ in (9) has been provided in [12]. A sufficient convergence condition on $\beta$ is derived in [12] by using the Gerschgorin circle theorem, which we describe in a lemma below.

Lemma 1. Suppose the parameter $\beta$ in (8) is chosen to be large enough satisfying

$$
\left|z_{l l}\right|+\frac{\beta}{\left|z_{l l}\right|} \geq\left|z_{l j}\right| \quad j \neq l, l=1,2
$$

Then the iterates in (9) converge to a fixed point for a sufficiently small step size $\mu$

$$
\boldsymbol{u}(\infty)=-\left(\beta \boldsymbol{I}+\boldsymbol{Z}_{d}^{H} \boldsymbol{Z}\right)^{-1} \boldsymbol{Z}_{d}^{H} \boldsymbol{d} .
$$

Furthermore, if $\beta=0$ is a solution to the set of inequalities in (10) and $\boldsymbol{Z}$ is nonsingular, the fixed point in (11) can be simplified as $\boldsymbol{u}(\infty)=-\boldsymbol{Z}^{-1} \boldsymbol{d}$, which provides the same noise reduction performance as the centralized controller.

The expression $\boldsymbol{u}(\infty)$ in (11) is in fact a function of the parameter $\beta$. We now study two extreme cases for $\beta$. When $\beta$ is sufficiently large, the matrix $\beta \boldsymbol{I}+\boldsymbol{Z}_{d}^{H} \boldsymbol{Z}$ will be dominated by $\beta \boldsymbol{I}$. As a result, $\boldsymbol{u}(\infty)$ can be approximated by $-\frac{1}{\beta} \boldsymbol{Z}_{d}^{H} \boldsymbol{d}$, which is far away from the desired optimal solution (3) or (4). This approximation suggests that a large parameter $\beta$ leads to significant degradation of the noise reduction performance. Conversely, $\beta=0$ leads to $\lim _{\beta \rightarrow 0} \boldsymbol{u}(\infty)=-\boldsymbol{Z}^{-1} \boldsymbol{d}$ when $Z$ is nonsingular, which is identical to (3) for the centralized controller. This suggests that a small $\beta$ value is favourable in terms of noise reduction performance. However, in general, the $\beta$ parameter cannot be chosen arbitrarily small for algorithmic stability.

The analysis above suggests that the conventional decentralized control method has to sacrifice the noise reduction performance for the purpose of guaranteed convergence. This limits the applicability of the conventional method to effectively control an ANC system.

\section{A NOVEL CONTROL METHOD BY SHAPING EIGENVALUES}

\section{A. Identification of two free parameters}

Let us revisit the convergence results for the conventional decentralized control method presented in Lemma 1. When $\beta=0$ and $\boldsymbol{Z}$ is nonsingular, the matrix $\boldsymbol{Z}_{d}$ is cancelled out in (11), leading to the same noise reduction performance as the centralied controller $\left(\left(\boldsymbol{u}(\infty)\right.\right.$ in $(11)$ coincides with $\boldsymbol{u}^{\star}$ in (3)). In other words, $Z_{d}$ does not contribute to the optimal solution $\boldsymbol{u}^{\star}$ in the above situation. If we replace $\boldsymbol{Z}_{d}$ with another diagonal matrix in (11), the optimal solution would not change.

We note that $\boldsymbol{Z}_{d}$ appears two times in (9): one coupled with the distortion $\boldsymbol{d}$ and the other coupled with $\boldsymbol{Z}$. The quantity $Z_{d}^{H} \boldsymbol{Z}$ appears inside the square bracket of (9). Therefore, $\boldsymbol{Z}_{d}$ has influence on the eigenvalues of the matrix product $\boldsymbol{Z}_{d}^{H} \boldsymbol{Z}$ which directly affects the stability of the steepest descent algorithm.

To briefly summarize, when $\beta=0$ and $Z$ is nonsingular, $Z_{d}$ has no contribution to the optimal solution $\boldsymbol{u}^{\star}$ in (3) while it affects the convergence of the steepest descent algorithm. Simulation results for the two-channel case in [12], [13] show 
that there exist situations where $\beta=0$ leads to algorithmic divergence due to the fact that the real part of at least one eigenvalue of $\boldsymbol{Z}_{d}^{H} \boldsymbol{Z}$ is negative.

One natural extension of the conventional decentralized control method is to replace the matrix $\boldsymbol{Z}_{d}$ in (8) and (9) by a diagonal matrix $\boldsymbol{C}=\operatorname{diag}\left[c_{11}, c_{22}\right]$, expressed as

$$
\begin{aligned}
\boldsymbol{u}(k+1) & =(1-\mu \beta) \boldsymbol{u}(k)-\mu \boldsymbol{C e}(k) \\
& =[\boldsymbol{I}-\mu(\beta \boldsymbol{I}+\boldsymbol{C} \boldsymbol{Z})] \boldsymbol{u}(k)-\mu \boldsymbol{C d},
\end{aligned}
$$

where $C$ remains to be specified. By doing so, we introduce two free parameters $c_{11}$ and $c_{22}$ to shape the eigenvalues of the matrix $\boldsymbol{C} \boldsymbol{Z}$ in (12) so that the parameter $\beta$ can either be set to zero or a small value to approach the same noise reduction performance as the centralized controller with guaranteed convergence. Specifically, our objective is to construct a matrix $C$ such that the real parts of the two eigenvalues of the matrix $\boldsymbol{C} \boldsymbol{Z}$ tend to be non-negative or positive. We will explain the steps to construct a proper matrix $C$ to achieve the above purpose in next subsection.

\section{B. On shaping the matrix eigenvalues}

In this subsection, we explain how to construct a proper diagonal matrix $C$ for the case that $Z$ is nonsingular. Construction of $C$ for $Z$ being singular is trivial, which we will briefly discuss in the end.

1) On defining a specific form for $C$ : We denote the two eigenvalues of the matrix product $\boldsymbol{C Z}$ in (12) as $\lambda_{1}=$ $\left|\lambda_{1}\right| e^{j \varphi_{1}}$ and $\lambda_{2}=\left|\lambda_{2}\right| e^{j \varphi_{2}}$ respectively. Without loss of generality, we let $\left|\varphi_{l}\right| \leq \pi, l=1,2$. We would like to construct a matrix $C$ such that the real parts of the two eigenvalues are non-negative or positive, expressed as

$$
\operatorname{Re}\left\{\lambda_{l}\right\} \geq 0 \quad l=1,2 .
$$

Or equivalently, the phases of the two eigenvalues satisfy $\left|\varphi_{l}\right| \leq \frac{\pi}{2}, l=1,2$. When the two strict inequalities hold in (13), one can set $\beta=0$ in (12) to reach the optimal input solution $\boldsymbol{u}^{\star}$ given by (3). On the other hand, if at least one equality holds in (13), one has to set $\beta>0$ to enable algorithmic convergence. Therefore, it is desirable to find a matrix $C$ that leads to two strict inequalities in (13).

We note that there might be many solutions for the matrix $C$ satisfying (13). In this paper, we let the matrix $C$ take a specific form as

$$
\boldsymbol{C}(\theta)=e^{j \theta}\left[\begin{array}{cc}
z_{11}^{*} & 0 \\
0 & z_{22}^{*}
\end{array}\right],
$$

where $C(\theta)$ indicates that $C$ is a function of the phase parameter $\theta$. Correspondingly, the two eigenvalues $\lambda_{1}$ and $\lambda_{2}$ of $\boldsymbol{C}(\theta) \boldsymbol{Z}$ are also functions of $\theta$, which we denote as $\lambda_{l}(\theta)=\left|\lambda_{l}(\theta)\right| e^{j \varphi_{l}(\theta)}, l=1,2$. It is straightforward that

$$
\begin{aligned}
\left|\lambda_{l}(\theta)\right| & =\left|\lambda_{l}(0)\right| & l & =1,2, \\
\varphi_{l}(\theta) & =\varphi_{l}(0)+\theta & l & =1,2 .
\end{aligned}
$$

The parameter $\theta$ has a linear contribution to the phases of the two eigenvalues while it does not affect the modulus of the two eigenvalues. Eqs. (14)-(16) simplify the procedure for

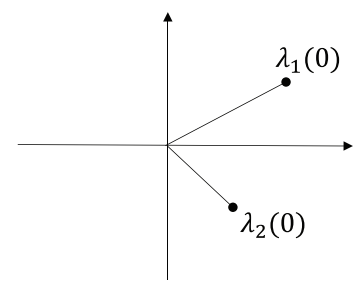

(a): Case 1

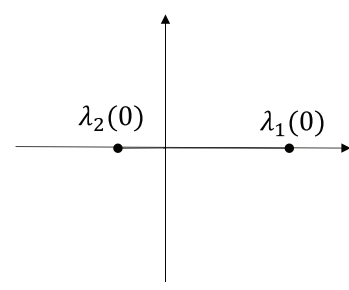

(c): Case 3

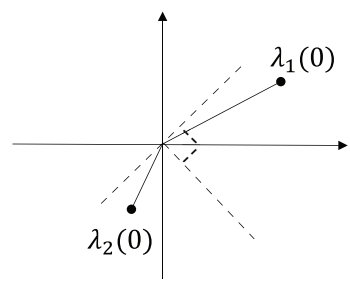

(b): Case 2

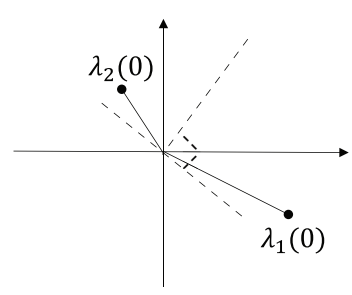

(d): Case 4
Fig. 2. Demonstration of the four relationships of the two eigenvalues $\lambda_{l}(0), l=1,2$.

constructing the matrix $C$ by reducing the number of parameters to only one real parameter $\theta$. The objective becomes to determine the parameter $\theta$ such that the phases of the two associated eigenvalues satisfy

$$
-\frac{\pi}{2} \leq \varphi_{l}(0)+\theta \leq \frac{\pi}{2} \quad l=1,2 .
$$

Next we study the relationship between the two eigenvalues $\lambda_{l}(\theta), l=1,2$, when $\theta=0$. With the expression (14), the matrix product $\boldsymbol{C}(0) \boldsymbol{Z}$ can be explicitly represented as

$$
\boldsymbol{C}(0) \boldsymbol{Z}=\left[\begin{array}{cc}
\left|z_{11}\right|^{2} & z_{11}^{*} z_{12} \\
z_{22}^{*} z_{21} & \left|z_{22}\right|^{2}
\end{array}\right] .
$$

By applying linear algebra to (18), the two eigenvalues $\lambda_{l}(0)$, $l=1,2$, exhibit a number of special properties, which we summarize in a lemma below:

Lemma 2. : When $\theta=0$, the two eigenvalues $\lambda_{1}(0)$ and $\lambda_{2}(0)$ of $\boldsymbol{C}(0) \boldsymbol{Z}$ satisfy

$$
\lambda_{1}(0)+\lambda_{2}(0)=\left|z_{11}\right|^{2}+\left|z_{22}\right|^{2},
$$

from which we conclude that at least one of the two eigenvalues $\lambda_{1}(0)$ and $\lambda_{2}(0)$ has positive real part. Further the imaginary parts of the two eigenvalues have the same magnitude but different signs, i.e., $\operatorname{Imag}\left(\lambda_{1}(0)\right)=-\operatorname{Imag}\left(\lambda_{2}(0)\right)$.

It is not difficult to conclude from Lemma 2 that the two eigenvalues $\lambda_{l}(0), l=1,2$, must fall into one of the following four cases (see Fig. 2 for illustration):

- Case 1: $-\frac{\pi}{2}<\varphi_{l}(0)<\frac{\pi}{2}, l=1,2$

- Case 2: $0<\varphi_{1}(0)<\frac{\pi}{2},-\pi \leq \varphi_{2}(0) \leq-\frac{\pi}{2}$

- Case 3: $\varphi_{1}(0)=0, \varphi_{2}(0)=\pi$

- Case 4: $-\frac{\pi}{2}<\varphi_{1}(0)<0, \frac{\pi}{2} \leq \varphi_{2}(0) \leq \pi$

As will be explained later, we need to construct different $\theta$ for different cases to satisfy the condition (17).

2) On computing $\theta$ for $\boldsymbol{C}(\theta)$ : So far we have characterized the relationship of the two eigenvalues $\lambda_{1}(\theta)$ and $\lambda_{2}(\theta)$ when $\theta=0$ into four cases as demonstrated in Fig. 2. The remaining 
task is to compute $\theta$ for each case to ensure the condition (17) hold. Geometrically speaking, the parameter $\theta$ rotates $\lambda_{1}(0)$ and $\lambda_{2}(0)$ in the complex domain around the origin. The degree of rotation depends on the locations of the two eigenvalues $\lambda_{1}(0)$ and $\lambda_{2}(0)$.

Now let us consider how to compute the rotation degree $\theta$ for the four cases separately. Case 1 states that the real parts of the two eigenvalues $\lambda_{1}(0)$ and $\lambda_{2}(0)$ are positive already. It is immediate that $\theta=0$ is one solution. That is there is no need to rotate the two eigenvalues any more. In this case, we can set $\beta=0$ in (12) to achieve the optimal noise reduction performance as the centralized controller.

In Case 2, the second eigenvalue $\lambda_{2}(0)$ is within the $3 \mathrm{rd}$ quadrant while the first eigenvalue $\lambda_{1}(0)$ is within the 1 st quadrant. In this case, it can be shown from Lemma 2 that

$$
\left|\varphi_{2}(0)\right|+\varphi_{1}(0)<\pi \text {. }
$$

The two eigenvalues cannot be on the same line which passes through the origin. Therefore, we are able to find a parameter $\theta$ so that after rotation both the two eigenvalues have positive real parts. One solution for $\theta$ can be computed by solving the following equation

$$
\left(\varphi_{1}(0)+\theta\right)=-\left(\varphi_{2}(0)+\theta\right)
$$

which implies that after rotation, the two phases $\varphi_{l}(\theta), l=$ 1,2 , have opposite signs. The expression for $\theta$ can be easily derived as

$$
\theta=\frac{1}{2}\left(\left|\varphi_{2}(0)\right|-\varphi_{1}(0)\right),
$$

Similarly to Case 1 , we can also set $\beta=0$ in (12).

Case 3 is a bit special in that the second eigenvalue $\lambda_{2}(0)$ takes a negative real number while the first eigenvalue $\lambda_{1}(0)$ takes a positive real number. In this case, it is obvious that $\theta=\frac{\pi}{2}$ or $\theta=-\frac{\pi}{2}$, is the optimal solution. After rotation, the resulting two eigenvalues are on the imaginary axis in the complex domain. To enable guaranteed convergence in (12), we have to set $\beta>0$ when running the iterates (12).

In Case 4, the second eigenvalue $\lambda_{2}(0)$ is within the 2 nd quadrant while the first eigenvalue $\lambda_{1}(0)$ is within the 4th quadrant. We can follow a similar analysis as for Case 2 . Solving (20) under the assumption that $\varphi_{1}(0)<0$ produces

$$
\theta=\frac{1}{2}\left(\left|\varphi_{1}(0)\right|-\varphi_{2}(0)\right) \text {. }
$$

Similarly to Case 1 and 2, we can also set $\beta=0$ in (12).

The analysis above for the four cases defines a procedure for properly shaping the eigenvalues of $\boldsymbol{C Z}$ in (12) to improve the noise reduction performance in comparison to [12], [13]. We summarize the results in a proposition below:

Proposition 1. Suppose the matrix $Z$ is nonsingular. If the two eigenvalues $\lambda_{1}(0)$ and $\lambda_{2}(0)$ fall into Cases 1,2 or 4 , a solution for the parameter $\theta$ can be computed to ensure the two eigenvalues of $\boldsymbol{C}(\theta) \boldsymbol{Z}$ have positive real parts. If the two eigenvalues $\lambda_{1}(0)$ and $\lambda_{2}(0)$ fall into Case $3, \theta=\frac{\pi}{2}$ or $\theta=-\frac{\pi}{2}$ ensures that the two eigenvalues of $C(\theta) Z$ have zero real parts.

We note that the solution for $\theta$ in Cases 1, 2 and 4 above
TABLE I

PROCEDURE OF THE PROPOSED DECENTRALIZED CONTROL METHOD

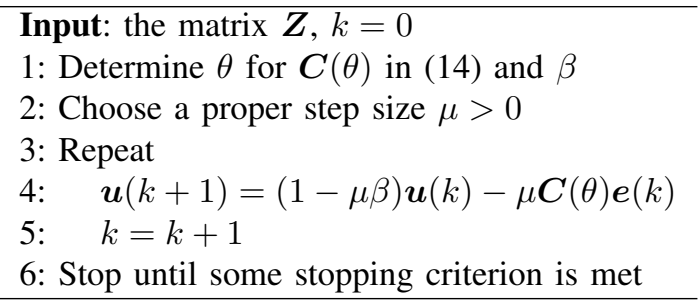

is not unique. One can also twist the parameter $\theta$ around each solution a bit, which would not change the sign of the eigenvalues. We can still set $\beta=0$ in (12) which allows the iterates in (12) to converge to $\boldsymbol{u}^{\star}$ in (3) for a sufficiently small step size $\mu$, leading to identical noise reduction performance as the centralized controller. This is similar to the single-channel research work [5], [6], [7] where only a rough estimation of the phase of secondary path is needed to guarantee the convergence of the steepest decent algorithm. It is worth noting that the phase parameter $\theta$ in (14) for the two-channel case requires to have a more accurate estimation than the phase of secondary path for the one-channel case.

Case 3 is different from the other three cases in that the two eigenvalues of $\boldsymbol{C}(\theta) \boldsymbol{Z}$ when $\theta=\frac{\pi}{2}$ or $\theta=-\frac{\pi}{2}$ have zero real parts. Therefore, one has to choose a positive weight factor $\beta>0$ in (12). By doing so, the resulting fixed point after convergence takes the form

$$
\boldsymbol{u}(\infty)=-e^{j \theta}\left(\beta \boldsymbol{I}+e^{j \theta} \boldsymbol{Z}_{d}^{H} \boldsymbol{Z}\right)^{-1} \boldsymbol{Z}_{d}^{H} \boldsymbol{d},
$$

where $\theta=\frac{\pi}{2}$ or $\theta=-\frac{\pi}{2}$. Eq. (23) has a different expression from the optimal solution in (3). A small $\beta$ parameter is favourable as $\boldsymbol{u}(\infty)$ would be a more accurate approximation of the optimal solution $\boldsymbol{u}^{\star}$. As a result, its noise reduction performance is nearly the same as that of the centralized controller.

Table 1 summarizes the procedure for implementing the proposed decentralized controller. The main difference with the conventional decentralized controller is that $C(\theta)$ is used before the error vector $e(k)$ instead of $Z_{d}^{H}$. The employment of $\boldsymbol{C}(\theta)$ enables the parameter $\beta$ to be set to either zero or a small positive number as discussed above.

Remark 1. The analysis for a singular matrix $\boldsymbol{Z}$ is similar to Case 1. One can easily show that the setup $C=Z_{d}^{H}$ would ensure that the two eigenvalues of $C Z$ are in the right-hand side of the complex domain, where one eigenvalue is zero. The fixed point after convergence takes the form

$$
\boldsymbol{u}(\infty)=-\left(\beta \boldsymbol{I}+\boldsymbol{Z}_{d}^{H} \boldsymbol{Z}\right)^{-1} \boldsymbol{Z}_{d}^{H} \boldsymbol{d},
$$

which is different from (4) that is obtained with the centralized controller.

\section{Simulations}

\section{A. Simulation setup}

We follow a similar simulation setup to that in [13] to evaluate our new decentralized control method. That is a 




(a)

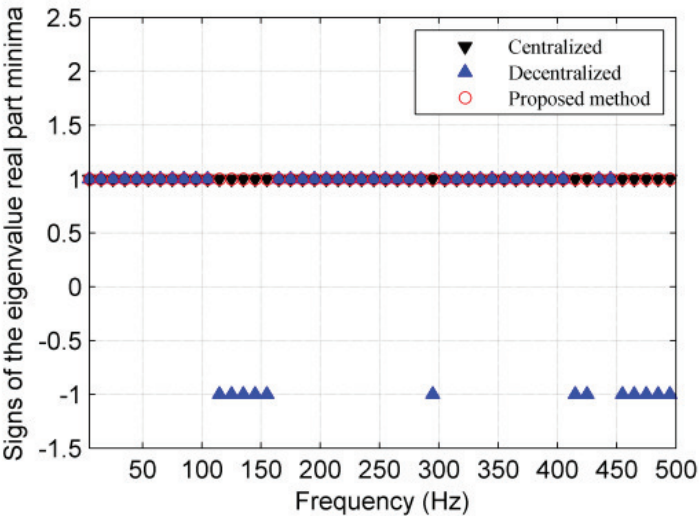

(b)

Fig. 4. The minima of the eigenvalues' real part and their signs. (a) the minima of the eigenvalues' real parts of $\boldsymbol{Z}^{H} \boldsymbol{Z}, \boldsymbol{Z}_{d}^{H} \boldsymbol{Z}$ and $\boldsymbol{C}(\theta) \boldsymbol{Z}$. (b) the signs of the minum of the eigenvalues' real part of $\boldsymbol{Z}^{H} \boldsymbol{Z}, \boldsymbol{Z}_{d}^{H} \boldsymbol{Z}$ and $\boldsymbol{C}(\theta) \boldsymbol{Z}$ ( "1" and "-1" stand for positive and negative respectively).

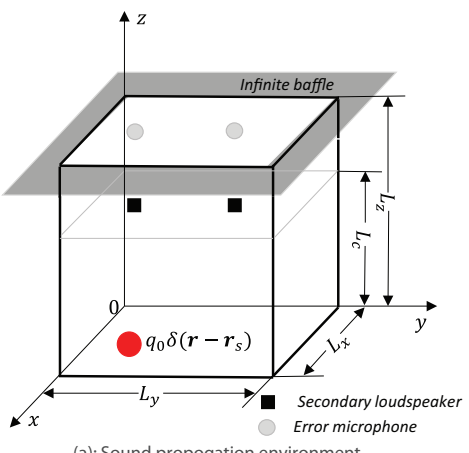

(a): Sound propogation environment

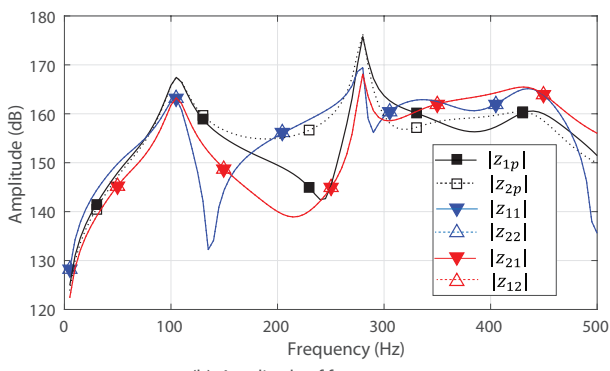

(b): Amplitude of frequency response

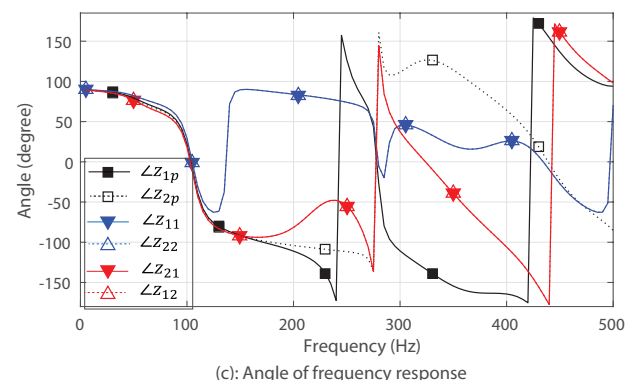

Fig. 3. Demonstration of the simulation setup. (a): A monopole sound source inside a rectangular enclosure with a baffled opening. (b): Amplitude of the frequency response $\left(z_{1 p}, z_{2 p}, z_{11}, z_{22}, z_{21}, z_{21}\right)$ in Fig. 1 below $500 \mathrm{~Hz}$. (c): Angle of the frequency response $\left(z_{1 p}, z_{2 p}, z_{11}, z_{22}, z_{21}, z_{21}\right)$ below $500 \mathrm{~Hz}$.

monopole sound source is located inside a rectangular enclosure with two channels of loudspeakers and microphones placed near a baffled opening, as demonstrated in Fig. 3 (a). The enclosure dimensions are chosen as $L_{x}=0.432 \mathrm{~m}$,

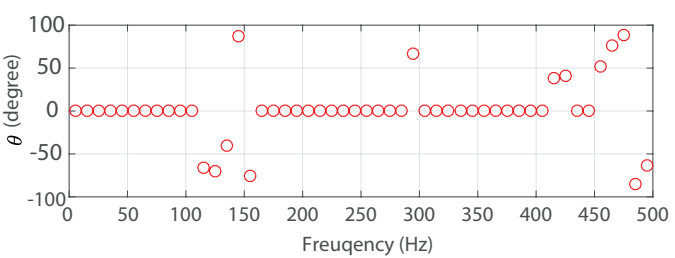

Fig. 5. $\theta$ value of the proposed method at different frequencies of Fig. 4.

$L_{y}=0.670 \mathrm{~m}$ and $L_{z}=0.598 \mathrm{~m}$. The primary source is placed at $\boldsymbol{r}_{s}=(0.12,0.12,0.16) \mathrm{m}$ with a strength of $q_{0}=1 \times 10^{-4} \mathrm{~m}^{3} / \mathrm{s}$. Two secondary loudspeakers are set at $(0.216,0.1675,0.498) \mathrm{m}$ and $(0.216,0.5025,0.498) \mathrm{m}$ with a distance of $0.335 \mathrm{~m}$ along the $\mathrm{y}$-axis. Correspondingly, two error microphones are set at $0.1 \mathrm{~m}$ above the secondary sources on the opening surface. The opening of the enclosure at $z=L_{z}$ is assumed to be embedded in an infinite baffle.

Fig. 3 (b) and (c) show the magnitudes and angles of the frequency responses (below $500 \mathrm{~Hz}$ ) of the acoustic paths from the primary and two secondary sources to the two error microphones, respectively. See Fig. 1 for the the physical correspondences of $\left(z_{1 p}, z_{2 p}, z_{11}, z_{22}, z_{21}, z_{21}\right)$. The simulation results in the two subplots were calculated using Matlab by following the mathematical expressions of the sound propagation model provided in $[13,16]$. It is seen from the subplots that $z_{11}=z_{22}$ and $z_{12}=z_{21}$, which is due to that fact that the simulation setup is symmetric w.r.t. the two ANC subsystems.

In the simulations, we tested three controlling methods: namely, the centralized controller, the conventional decentralized controller considered in [12], [13], and our newly proposed decentralized controller. We focused on the convergence properties of the three methods by setting the weighting factor $\beta=0$ which leads to the maximum noise reduction performance. The noise reduction performance at each frequency is evaluated in terms of the sound pressure level (SPL) at the two error microphones, given by

$$
\mathrm{SPL}=\frac{1}{2} \sum_{l=1}^{2} 20 \log _{10}\left[\operatorname{abs}\left(e_{l}\right) /\left(2 \times 10^{-5}\right)\right],
$$


where $e_{l}, l=1,2$, are the sound pressure error signals, and the operation $\operatorname{abs}(\cdot)$ computes the absolute value.

\section{B. On the stability of the three methods}

As discussed in Section 4, the stability of the proposed method is guaranteed only when the real parts of the two eigenvalues of $\boldsymbol{C}(\theta) \boldsymbol{Z}$ are positive for each frequency bin. Similarly, the stabilities of the centralized controller and the conventional decentralized controller (referred to as "decentralized") are determined by the eigenvalues' real parts of the two matrices $\boldsymbol{Z}^{H} \boldsymbol{Z}$ and $\boldsymbol{Z}_{d}^{H} \boldsymbol{Z}$, respectively. We therefore denote the minima of the eigenvalues' real parts for $\boldsymbol{Z}^{H} \boldsymbol{Z}$, $\boldsymbol{Z}_{d}^{H} \boldsymbol{Z}$ and $\boldsymbol{C}(\theta) \boldsymbol{Z}$ as $\lambda_{R e, m i n}^{\text {cent }}, \lambda_{R e, \text { min }}^{\text {decent }}$ and $\lambda_{R e, m i n}^{\text {prop }}$. Fig. 4 shows the distribution of $\lambda_{R e, m i n}^{c e n t}, \lambda_{R e, m i n}^{\text {decent }}$ and $\lambda_{R e, \text { min }}^{\text {prop }}$ below $500 \mathrm{~Hz}$. Furthermore, Fig. 5 displays the $\theta$ value of the proposed method at different frequencies of Fig. 4.

It is clear from Fig. 4 that our proposed method manages to shape the eigenvalues of $\boldsymbol{C}(\theta) \boldsymbol{Z}$ by choosing proper $\theta$ value as illustrated in Fig. 5 over all the considered frequency bins, ensuring that the real parts of all the eigenvalues are positive. As a result, the proposed control method is able to achieve the same noise reduction performance as the centralized one. By contrast, the conventional decentralized method diverges at several frequency bins where the minima of the eigenvalues' real part are negative (i.e., $\lambda_{\text {Re,min }}^{\text {decent }}<0$ ). To fix the convergence issue in this situation, the noise reduction performance of the conventional decentralized method has to be sacrificed by imposing constraints on the magnitude of the input to the loudspeakers (i.e., choosing proper weighting factor $\beta>0$ ). The proposed method has an advantage over the conventional decentralized method by fixing its convergence issue.

\section{Comparison of convergence speeds}

Besides the guaranteed convergence of the proposed method, it is also of interest to study its convergence speed in comparison with the other two methods. Fig. 6 (a) and (b) display the iteration behaviours of the three methods in terms of the sound pressure level at two frequencies $130 \mathrm{~Hz}$ and $200 \mathrm{~Hz}$, respectively. Two step sizes $\mu_{1}=1 \times 10^{-7}$ and $\mu_{2}=1 \times 10^{-6}$ were tested.

We first consider the results in Fig. 6 (a) for $130 \mathrm{~Hz}$. One observes that when the smaller step size $\mu_{1}$ is adopted, the proposed method converges relatively slowly compared to the centralized method while the conventional decentralized method fails to converge. For the case of larger step size $\mu_{2}$, both the proposed and the conventional decentralized method diverge while only the centralized method converges.

The behaviors of the three methods in Fig. 6 (a) can be explained by the eigenvalue properties as shown in Fig. 4 (a). It is clear that at frequency $130 \mathrm{~Hz}, \lambda_{R e, \text { min }}^{\text {cent }}>\lambda_{R e, \text { min }}^{\text {prop }}>$ $0>\lambda_{R e, m i n}^{\text {decent }}$. As a result, the upper bound of step size for the centralized method is greater than that for the proposed method, explaining divergence of the proposed method at $\mu_{2}$. The divergence of the conventional decentralized method at the two step sizes can be explained by the fact that at frequency $130 \mathrm{~Hz}$, one of its matrix eigenvalues has a negative real part (i.e., $0>\lambda_{R e, m i n}^{\text {decent }}$ ). This confirms that the proposed

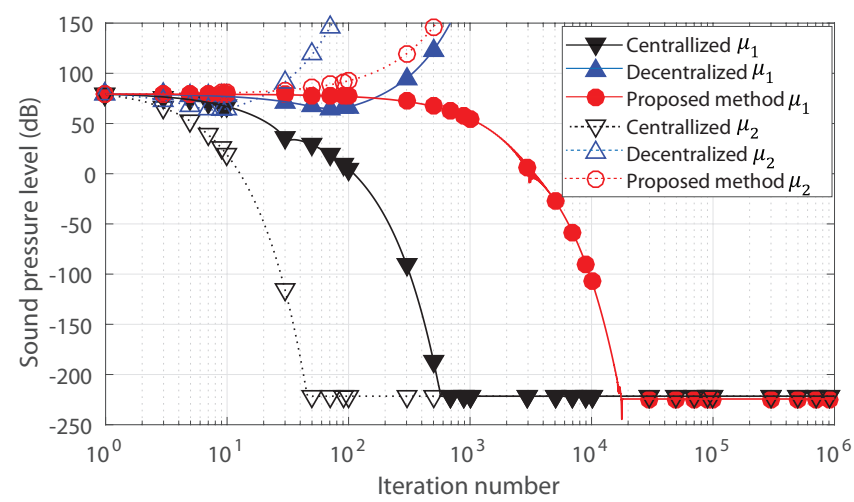

(a): Evaluation at $130 \mathrm{~Hz}$

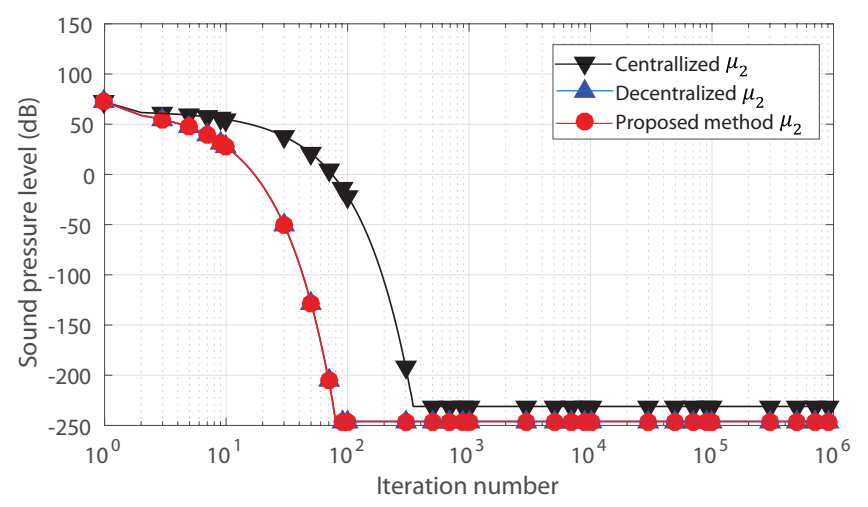

(b): Evaluation at $200 \mathrm{~Hz}$

Fig. 6. Convergence curves of the three methods with weighting factors of zero at $130 \mathrm{~Hz}$ and $200 \mathrm{~Hz}$, where $\mu_{1}=1 \times 10^{-7}$ and $\mu_{2}=1 \times 10^{-6}$.

method is effective in terms of stability improvement over the conventional decentralized method.

Fig. 6 (b) shows the convergence behaviours of the three methods at $200 \mathrm{~Hz}$ by using the larger stepsize $\mu_{2}$. It is clear that the proposed method has identical convergence as the conventional decentralized method, which is due to the fact that $\theta=0$ as shown in Fig. 5. Furthermore, the two methods exhibit considerably faster convergence than the centralized controller. The above property is closely related to the eigenvalue properties provided in Fig. 4 (a). It is immediate from the figure that at $200 \mathrm{~Hz}$, there is $\lambda_{R e, \min }^{\text {prop }}=\lambda_{R e, \text { min }}^{\text {decent }}>$ $\lambda_{R e, \min }^{\text {cent }}>0$. The slower convergence of the centralized method is due to the smaller parameter $\lambda_{R e, \min }^{c e n t}$.

The results from both Fig. 6 (a) and (b) suggest that for a fixed stepsize, a method with a larger value of $\lambda_{R e, \min }$ leads to faster convergence speed. In addition, the centralized controller does not always have faster convergence speed than the other two methods under the same stepsize.

\section{Upper bounds of the step size}

The results of Fig. 6 (a) suggest that different methods may have different upper bounds of step size to ensure algorithmic convergence. In this subsection, we consider computing the upper bounds of step size for the three methods over the frequencies below $500 \mathrm{~Hz}$.

For each frequency bin, we tested an array of step sizes for each method. The array was constructed as $1.1^{m-1} \times 10^{-9}$, 


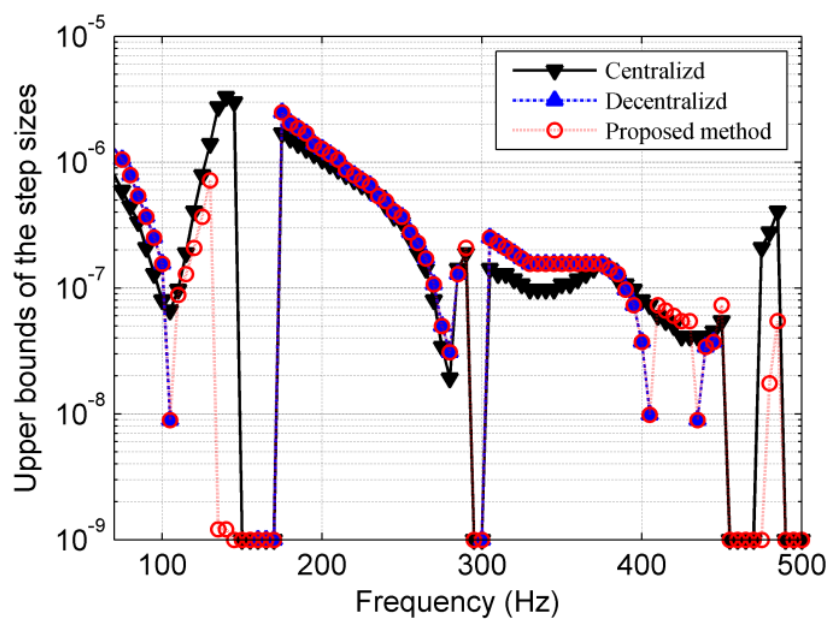

Fig. 7. Upper bound of the step size to ensure convergence for the 2-channel systems with weighting factors of zero.

where $m=1,2, \ldots$, is the array index. Each upper bound in the figure was obtained when its next tested step size in the array leads to algorithmic divergence. We point out that for an array index $m$, the tested step size for each method may lead to a different speed of convergence as illustrated by Fig. 6 .

Fig. 7 shows the computed upper bounds for the three methods. The results for the conventional decentralized method are missing for a set of frequency bins due to the fact that it always diverges for any small step size at these frequencies, which is consistent with the results of Fig. 4.

It is observed from the figure that at some frequencies, the upper bounds of the centralized method are higher than those of the other two methods while at other frequencies, an opposite trend can be seen. In other words, the centralized method does not always produce higher upper bounds. The above property is in line with the observation that the centralized method does not always have fastest convergence speed as discussed in Subsection V-C.

We also notice that for a few frequencies (around $130 \mathrm{~Hz}$ ), the upper bounds of the proposed method are close to $10^{-9}$ while those of the centralized method are around $10^{-6}$. The above noticeable difference is due to the fact the two channels are deeply coupled at those frequencies. Consequently, the two eigenvalues of $\boldsymbol{C}(\theta) \boldsymbol{Z}$ of the proposed method at each frequency have a large phase difference (close to 180 degree) in comparison to the centralized method, leading to a small $\lambda_{\text {Re,min }}^{\text {prop }}>0$ (see Fig. 2 and Fig. 4).

\section{CONCLUSions AND FutURE WORK}

In this paper, we have proposed a new adaptive decentralized control method for a two-channel ANC system in the frequency domain. The method exploits one real parameter to properly shape the two eigenvalues of a constructed matrix which results from the two-channel secondary paths for each frequency bin. By doing so, the adaptive control method is able to improve its convergence stability without sacrificing the noise reduction performance in comparison with the conventional decentralized controller. Extensive simulations have been conducted, which confirm the effectiveness of the proposed method.

One future research direction would be to consider the possibility of extending the current work to multi-channel ANC. That is to identify and tune the free parameters for multi-channel case to enable effective decentralized control. In general, the coupling effect of the multi-channel ANC increases quadratically as the channel number increases while the number of free parameters increases linearly under decentralized control. The resulting decentralized control method may not be able to achieve the same noise reduction performance as the centralized controller. To further improve the performance, one solution would be to allow neighboring ANC subsystems share their microphone error signals to enhance controllability.

One can also extend the current work to address broadband noise for two-channel decentralized control. To do so, one can first follow the standard approach by dividing the noise into several sub-bands, and then treat each sub-band noise separately using the proposed method. The effectiveness of the proposed method depends on the the phase variation range of each sub-band. In general, the smaller the range is, the larger the searching space of the proposed method for finding the optimal direction of the steepest decent method. Correspondingly, better noise reduction performance can be obtained at the cost of high computational complexity due to a large number of sub-bands.

\section{ACKNOWLEDGEMENTS}

This research was supported under the Australian Research Council's Linkage Project funding scheme (LP140100987, Integrated Passive and Active Control of Humming Noise from KCGMs Haul Trucks).

\section{REFERENCES}

[1] S. Kuo, S. Mitra, and W.-S. Gan, "Active noise control system for headphone applications," IEEE Trans. Control Systems Technology, vol. 14, no. 2, pp. 331-335, 2006.

[2] C. Chen, T.-D. Chiueh, and J.-H. Chen, "Active cancellation system of acoustic noise in MR imaging," IEEE Trans. Biomedical Engineering, vol. 46, no. 2, pp. 186-191, 1999.

[3] M. D. Diego, A. Gonzalez, M. Ferrer, and G. Pinero, "An adaptive algorithms comparison for real multichannel active noise control," in 12th European Signal Processing Conference, 2004.

[4] T. Kosaka, S. J. Elliott, and C. C. Boucher, "A Novel Frequency Domain Filtered-X LMS Algorithm for Active Noise Reduction," in IEEE International Conference on Acoustics, Speech, and Signal Processing (ICASSP), 1997, pp. 403-406.

[5] D. Zhou and V. DeBrunner, "A new active noise control algorithm that requires no secondary path identification based on SPR property," IEEE Trans. Signal Processing, vol. 55, no. 5, pp. 1719-1729, 2007.

[6] M. Wu, G. Chen, and X. Qiu, "An Improved Active Noise Control Algorithm Without Secondary Path Identification Based on the Frequency-Domain Subband Architecture," IEEE Trans. Audio, Speech, and Language Processing, vol. 16, no. 8, pp. 1409-1419, 2008.

[7] M. Gao, J. Lu, and X. Qiu, "A simplified subband ANC Algorithm Without Secondary Path Modeling," IEEE Trans. Audio, Speech, and Language Processing, vol. 24, no. 7, pp. 1164-1174, 2016.

[8] T. Murao, C. Shi, W. S. Gan, and M. Nishimura, "Mixed-error approach for multi-channel active noise control of open windows," Applied Acoustics, vol. 127, pp. 305-315, 2017.

[9] M. Ferrer, M. Diego, G. Pinero, and A. Gonzalez, "Active Noise Control over Adaptive Distributed Networks," Signal Processing, vol. 107, pp. 82-95, 2015. 
[10] C. Antonanzas, M. Ferrer, A. Gonzalez, M. D. Diego, and G. Pinero, "Diffusion Algorithm for Active Noise Control in Distributed Networks," in ICSV22, 2015.

[11] Y. Chu and C.-M. Mak, "Diffusion Control for Multi-Channel ANC Systems Using Filtered-X Algorithms," in 24th International Congress on Sound and Vibration, 2017.

[12] S. J. Elliott and C. C. Boucher, "Interaction Between Multiple Feedforward Active Control Systems," IEEE Trans. Speech and Audio Processing, vol. 2, no. 4, pp. 521-530, 1994.

[13] J. Tao, S. Wang, X. Qiu, and J. Pan, "Performance of an independent planar virtual sound barrier at the opening of a rectangular enclosure," Applied Acoustics, vol. 105, pp. 215-223, 2016. 